\title{
Dauerhaft exzellent? Personalrekrutierung und Modelle nachhaltiger Karriereentwicklung im Kontext der Exzellenzinitiative
}

\author{
Michael Sondermann, Sebastian Bukow, Dagmar Simon
}

Die Exzellenzinitiative hat wie kaum ein anderes Förderprogramm Bewegung in die deutsche Wissenschaftslandschaft gebracht. Gleichzeitig wird kein anderes Förderprogramm derart kontrovers diskutiert. Der nachfolgende Beitrag fokussiert auf die wissenschaftlichen Akteure, denn schließlich wurden innerhalb kurzer Zeit über 4.000 neue Stellen für Doktoranden, Postdocs und Professoren geschaffen und besetzt. Dies bietet vielfältige Chancen für neue Wege in der Personalrekrutierung und -entwicklung, birgt aber auch Risiken und Konfliktpotenziale. Offen ist zudem, ob die eingeführten Veränderungen über die Exzellenzinitiative hinaus wirken.

\section{Einleitung}

Dem Dreikönigstag 2004 kommt besondere Bedeutung für die deutsche Hochschulpolitik zu: An diesem Tag wurde eine Idee in die Welt gesetzt, die später in der Exzellenzinitiative münden sollte. Die SPD forderte nach ihrer Klausurtagung in Weimar am 4./5. Januar in den „Weimarer Leitlinien Innovation“, man müsse in Deutschland „die Struktur der Hochschullandschaft so verändern, dass sich Spitzenhochschulen und Forschungszentren etablieren, die auch weltweit in der ersten Liga mitspielen und mit internationalen Spitzenhochschulen wie Harvard und Stanford konkurrieren können“ (SPD 2004). Zwar reicht die Diskussion über Spitzenförderung an deutschen Hochschulen weiter zurück, dennoch hat die Exzellenzinitiative ein ungewöhnlich lautes Echo in Politik, Medien und nicht zuletzt der Wissenschaft selbst ausgelöst (Hornbostel et al. 2008; Münch 2007; Hartmann 2006; Markschies 2007; Simon/Knie 2007). Die politischen Entscheidungsträger sind für ihre Ideen gelobt worden, mussten aber auch deutliche Kritik hinnehmen; von „staatlichen Regelungsphantasien" war die Rede, ausgelöst durch einen „Harvard-Komplex“, unter dem die Bundesregierung leide (von Münch 2005). Auch in der Politik selbst verlief die weitere Aushandlung der Ausgangsidee hin zur im Juni 2005 zwischen Bund und Ländern vereinbarten Exzellenzinitiative nicht ohne Reibungsverluste (Pasternack 2008). Herausgekommen ist schließlich ein Programm, das mit seinen drei Förderlinien (Graduiertenschulen,
Exzellenzcluster, Zukunftskonzepte) von Spitzenförderung über Nachwuchsausbildung, Interdisziplinarität, internationaler Konkurrenzfähigkeit, Zusammenarbeit mit der außeruniversitären Forschung bis hin zu Strukturveränderungen und Profilbildung eine (über-)große Fülle an wissenschaftspolitischen Handlungsfeldern adressiert, die in den Debatten der letzten zwei Dekaden immer wieder auf der Agenda standen. Die Exzellenzinitiative hat dabei wie ein Brennglas auf die Probleme der Karrierebedingungen, -entwicklung und -systeme im deutschen Wissenschaftssystem aufmerksam gemacht. Zugleich hat sie ein variantenreiches Experimentierfeld eröffnet (Sondermann et al. 2008). Der vorliegende Beitrag beleuchtet Maßnahmen und bereits erkennbare Wirkungen im Bereich des Aufbaus und der Weiterentwicklung nachhaltiger Karrierewege für den wissenschaftlichen Nachwuchs im Kontext der Exzellenzinitiative. Der Blick richtet sich insbesondere auf Modelle einer Karriereentwicklung, die die Übergänge von Karriere- bzw. Qualifikationsstufen im Visier hat und Tenure-Optionen, also kalkulierbare, wenn auch nicht zugleich „verbriefte“ Beschäftigungsperspektiven anbietet. Empirisch stützt sich der Beitrag auf Daten des Instituts für Forschungsinformation und Qualitätssicherung (iFQ). ${ }^{1}$

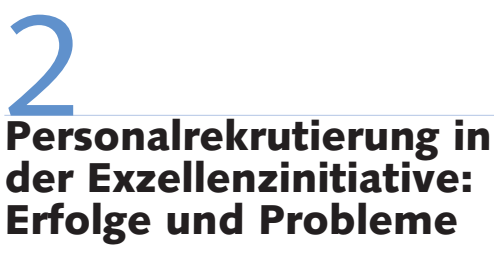

Eine wissenschaftliche Karriere stellt sich in Deutschland als ein unsicheres Unter- fangen dar: Vor allem die Übergänge von einer Qualifikations- und Karrierestufe zur nächsten bergen ein hohes Maß an Unsicherheit (Enders 1996; Janson et al. 2007).

\footnotetext{
1 Das iFQ betreibt seit Mitte 2009 eine Untersu chung, die sich mit Wirkungen der Initiative auf die Rekrutierung wissenschaftlichen Spitzenpersonals auseinandersetzt. Bis Februar 2010 wurden insgesamt 40 Interviews mit Sprechern von Graduiertenschulen und Exzellenzclustern sowie Universitätskanzlern und -rektoren bzw. -präsidenten geführt.
}

Michael Sondermann, Dipl.-Geograph, ist wissenschaftlicher Mitarbeiter am Institut für Forschungsinformation und Qualitätssicherung (iFQ) und koordiniert hier ein Monitoring der Exzellenzinitiative.

Arbeitsschwerpunkte: Evaluationsforschung, Effekte von Cluster- und Netzwerkförderprogrammen. e-mail: sondermann@forschungsinfo.de Sebastian Bukow, Dipl.-Soziologe, ist wissenschaftlicher Mitarbeiter am iFQ. Arbeitsschwerpunkte: Politisches System der BRD, Parteien- und Parteiensystemforschung, Organisationsforschung und Hochschulpolitik. e-mail: bukow@forschungsinfo.de Dagmar Simon, Dr. rer. pol., leitet die Forschungsgruppe Wissenschaftspolitik am Wissenschaftszentrum Berlin für Sozialforschung (WZB). Von 2006 - 2007 war sie für die wissenschaftliche Koordination des iFQ verantwortlich und arbeitete im Projekt "Monitoring der Exzellenzinitiative" mit. Arbeitsschwerpunkte: Wissenschafts-, Organisations- und Evaluationsforschung sowie Gender Studies. e-mail:dsimon@wzb.eu 
Hinzu kommt die stark hierarchisch geprägte Struktur des deutschen Hochschulsystems, die sich beispielsweise in der Doktorandenausbildung zeigt (Buchholz et al. 2009; Gülker/Böhmer 2009) und der unter anderem mit dem Aufbau strukturierter Promotionsprogramme begegnet wird. Auf der nächsten Karrierestufe - in der Postdoc-Phase - sind die Perspektiven noch unsicherer, zumal die „natürliche Endposition" Professur in Deutschland jedenfalls gemessen an der Nachfrage rar ist. Aktuell fehlen damit mindestens zwei Dinge: Erstens gibt es noch immer keine tragfähigen Karrieremodelle zu einer zielgerichteten Personalentwicklung insbesondere für die Postdoc-Phase. Zweitens fehlt es an attraktiven Folgepositionen für das Heer der befristet beschäftigten Postdocs im deutschen Wissenschaftssystem.

Was trägt nun die Exzellenzinitiative zur Problemlösung bei? Welche neuen Problemlagen entstehen möglicherweise? Die Exzellenzinitiative berührt in allen Förderlinien essentielle Aspekte von Nachwuchsausbildung und Karriereentwicklung in Wissenschaft und Forschung. Graduiertenschulen sollen die Modelle der strukturierten Promotion substanziell voranbringen und eine „Doktorandenkultur“ an den geförderten Hochschulen erzeugen. Exzellenzcluster entpuppen sich als wahre Jobmaschinen für den wissenschaftlichen Nachwuchs und bauen - ein interessantes Ergebnis - ebenfalls in erheblichem Maße Elemente einer strukturierten Doktorandenausbildung auf (Sondermann et al. 2008). Die Zukunftskonzepte, sicherlich die Programmlinie mit dem buntesten Maßnahmenstrauß, sehen ebenfalls Fördertöpfe zur Verbesserung der beruflichen Situation und der Karriereperspektiven von Nachwuchswissenschaftlerinnen und -wissenschaftlern vor (DFG/WR 2008). Dabei wird in allen drei Förderlinien mit Konzepten früher Selbstständigkeit (Nachwuchsforschergruppen), Verstetigungsoptionen, Dual-Career-Programmen, etc. gearbeitet.

Der Bund und die Länder stellen im Zeitraum von 2006 bis 2012 insgesamt 1,9 Mrd. Euro Fördermittel zur Verfügung. Von 2012 bis 2017 werden noch einmal 2,7 Mrd. Euro bereitgestellt (Bundesanzeiger 2009). Ein Großteil der Mittel wird genutzt, um wissenschaftliches Personal einzustellen. Tabelle 1 zeigt den Rekrutierungsstand in allen Förderlinien zum Frühjahr 2009. Dargestellt ist die Anzahl der Personen, deren Positionen (Stelle, Stipendium)

Tabelle 1: Rekrutierung wissenschaftlichen Personals aus Mitteln der Exzellenzinitiative, Stand Februar 2009

\begin{tabular}{|c|c|c|c|c|}
\hline & & $\begin{array}{c}\text { Stand } \\
\text { Februar } 2009 \\
\text { (absolut) }\end{array}$ & $\begin{array}{c}\text { davon } \\
\text { weiblich } \\
\text { (in \%) }\end{array}$ & $\begin{array}{l}\text { davon } \\
\text { aus dem } \\
\text { Ausland* } \\
\text { (in \%) }\end{array}$ \\
\hline \multirow{4}{*}{$\begin{array}{l}\text { Graduiertenschulen } \\
\text { (1. Förderlinie) }\end{array}$} & Professoren & 34 & 29,4 & 11,8 \\
\hline & davon Juniorprof. & 16 & 43,8 & 25,0 \\
\hline & $\begin{array}{l}\text { Promovierte Nachwuchswiss. } \\
\text { (inkl. Nachwuchsgruppenleiter) }\end{array}$ & 75 & 42,7 & 26,7 \\
\hline & Doktoranden (inkl. Fast-Track) & 901 & 43,0 & 33,3 \\
\hline \multirow{4}{*}{$\begin{array}{l}\text { Exzellenzcluster } \\
\text { (2. Förderlinie) }\end{array}$} & Professoren & 147 & 27,2 & 28,6 \\
\hline & davon Juniorprof. & 55 & 34,5 & 38,2 \\
\hline & $\begin{array}{l}\text { Promovierte Nachwuchswiss. } \\
\text { (inkl. Nachwuchsgruppenleiter) }\end{array}$ & 588 & 32,8 & 22,8 \\
\hline & Doktoranden (inkl. Fast-Track) & 1323 & 34,6 & 17,5 \\
\hline \multirow{3}{*}{$\begin{array}{l}\text { Zukunftskonzepte } \\
\text { (3. Förderlinie) }\end{array}$} & Professoren & 145 & 31,7 & 27,8 \\
\hline & davon Juniorprof. & 28 & 57,1 & 48,1 \\
\hline & $\begin{array}{l}\text { alle Nachwuchswissenschaftler } \\
\text { (Nachwuchsgruppenleiter, Postdocs } \\
\text { und Doktoranden inkl. Fast-Track) }\end{array}$ & 844 & 40,6 & 17,5 \\
\hline \multicolumn{5}{|c|}{$\begin{array}{l}\text { *Für Graduiertenschulen und Exzellenzcluster liegen in allen Personalkategorien für mehr als } 90 \% \text { der Fälle Angaben zum } \\
\text { Herkunftsland vor. Für Zukunftskonzepte liegen für die promovierten Nachwuchswissenschaftler und Doktoranden solche } \\
\text { Angaben mehrheitlich nicht vor. Berechnung bei Zukunftskonzepten nur für Personen mit Angaben zum Herkunftsland. }\end{array}$} \\
\hline \multicolumn{3}{|c|}{$\begin{array}{l}\text { Quelle: iFQ-Stammdatenerhebung bei Graduiertenschulen und Exzellenzclustern } \\
\text { im Februar 2009, Wissenschaftsrat Erhebung bei Zukunftskonzepten im Februar } 2009\end{array}$} & 18 & ILUNGEN \\
\hline
\end{tabular}

überwiegend (d.h. zu mindestens $50 \%$ ) aus Mitteln der Exzellenzinitiative finanziert werden.

Der überwiegende Anteil der Positionen wurde auf der Nachwuchsebene geschaffen. In Graduiertenschulen und Exzellenzclustern (für Zukunftskonzepte liegen keine Detaildaten vor) sind 72,5\% der insgesamt 3068 bis Frühjahr 2009 aus „Exzellenzgeldern“ rekrutierten Personen Promovierende, 21,6\% sind Postdocs und $5,9 \%$ haben eine Professur. Die „Personalintensität" der einzelnen Projekte ist sehr unterschiedlich. Das liegt vor allem an der sehr flexiblen Gestaltung der Exzellenzinitiative - einschließlich der Möglichkeit, Sach- und Personalmittel umzuwidmen. Entsprechend variantenreich sind die Projekte: Von Exzellenzclustern, die einen Großteil der Fördergelder für neue Professuren verwenden, bis zu solchen, die die Mittel vor allem für die Förderung des wissenschaftlichen Nachwuchses einsetzen. Bei Graduiertenschulen lassen sich ähnliche Unterschiede zeigen, insbesondere bei der Anzahl der jährlich zu vergebenden Promotionsstipendien.

Dass diese beachtliche Zahl an Personen - insgesamt über 4000 (inkl. der Zukunftskonzepte) - binnen kurzer Zeit eingestellt werden konnte, ist sicherlich ein sichtbares Erfolgszeichen der Exzellenzinitiative. Aus einer Systemperspektive bedarf es für eine Gesamtbewertung gleichwohl weiterer Untersuchungen, die auch die Auswirkungen auf nicht exzellenzge- förderte Hochschulen einbeziehen. An den geförderten Hochschulen scheint sich der betriebene Aufwand zu lohnen. Eine Onlinebefragung des iFQ bei Exzellenzprojekten im Mai 2008 zeigt: Die Mitglieder der Schulen und Cluster (Zukunftskonzepte wurden vom iFQ nicht befragt) sind ganz überwiegend der Ansicht, dass die „Wunschkandidatinnen und -kandidaten“ gewonnen werden konnten - und zwar sowohl was Promovierende und Postdocs als auch die bis dato berufenen Professoren anbelangt (Sondermann et al. 2008). Gleichzeitig wurden aber auch gravierende Probleme deutlich. Aus Sicht der Mitglieder von Exzellenzclustern waren ein Mangel an geeigneten Kandidaten (23,4\% der Antwortenden), das als nicht wettbewerbsfähig empfundene Gehaltsniveau im deutschen Wissenschaftssystem (16,2\%) und die Befristung der ausgeschriebenen Positionen (14,0 \%) die Hauptschwierigkeiten bei der Personalgewinnung (ebd.). Verschärfend hinzu kommt der Zeitdruck, unter dem die Projekte stehen: In den Jahren 2011/12 steht die Begutachtung der Fortsetzungsanträge für die nächste Runde der Exzellenzinitiative an. Die Sprecher der Graduiertenschulen und Exzellenzcluster gehen sicher zu Recht davon aus, dass ein Kriterium für die erreichte „Exzellenz“ ihrer Projekte die Qualität der Personalrekrutierung sein wird. Entsprechend hoch ist der Aufwand, der hierfür betrieben wird. Bereits in den Förderanträgen ist sehr deutlich formuliert, dass 
die Antragsteller hier an unterschiedlichen Punkten beabsichtigen, „eingetretene“ Pfade bei der Personalgewinnung, aber auch bei der konkreten Ausgestaltung der Positionen zu verlassen.

\section{Karrierepfade}

\subsection{FAST TRACK}

Die konkrete Umsetzung dieser selbst gesetzten Zielvorgabe basiert dabei auf Elementen, die per se zwar nicht immer neu sind, in der nun verfolgten Breite aber durchaus eine andere Dimension für das deutsche Wissenschaftssystem darstellen können. Dabei nehmen US-amerikanische Modelle eine wichtige Orientierungsfunktion ein. Sichtbar wird dieses besonders im Bereich der Nachwuchsförderung. Für die Startphase der wissenschaftlichen Karriere werden durch die Einführung sogenannter „Fast Track“-Modelle neue Pfade geschaffen. Die Idee ist, herausragenden Bachelorstudenten ohne Masterabschluss direkt den Übergang in eine strukturierte Promotion zu ermöglichen, nicht zuletzt, um Ausbildungszeiten zu verkürzen. Solche Modelle stoßen aber im deutschen Hochschulsystem gleich in mehrfacher Hinsicht auf Probleme. Zum einen sind sie in aller Regel neu, sodass die zugrunde liegenden Studien- und Promotionsordnungen angepasst werden müssen - was im gegenwärtig (wieder) Bologna-gestressten System kein leichtes Unterfangen ist. Zum anderen kommen auf die Promovierten im weiteren Verlauf ihrer (wissenschaftlichen) Karriere dienstrechtliche Probleme zu: Um - beispielsweise als Professor - im höheren Dienst verbeamtet zu werden, muss ein Masterabschluss vorliegen. Die Realität in den Graduiertenschulen - und die betrifft es hauptsächlich - zeigt sich also als Kombimodell: Schneller Einstieg in die Promotion und in der Startphase zusätzlich ein Masterabschluss. Auch dieses erfordert Koordinierungsaufwand und gegebenenfalls Flexibilität in der Interpretation und Ausgestaltung vorhandener Studienordnungen, was durchaus Konfliktpotenzial birgt. Für den kurzen Abschnitt des Masterabschlusses ergeben sich überdies Finanzierungsprobleme, denn Exzellenzmittel dürfen nicht für Maßnahmen im Bereich der Lehre genutzt werden.

\subsection{POSTDOCS ON TRACK?}

Für Postdocs werden vor allem in den Exzellenzclustern Karrieremodelle eingerichtet, die gezielt auf die spätere Berufung auf eine Professur vorbereiten sollen. Besonders qualifizierte Wissenschaftler erhalten die Chance, innerhalb der Cluster ein eigenes Forscherteam aufzubauen. Dieser Karriereweg macht seit Längerem Schule: Die Max-Planck-Gesellschaft fördert seit 1969 selbstständige Nachwuchsgruppen, die Helmholtz-Gemeinschaft seit 2002, die Deutsche Forschungsgemeinschaft (DFG) hat 1999 das Emmy Noether-Programm aufgelegt, etc. Wie bei diesen Programmen erhalten auch die Postdocs in den Exzellenzclustern eine attraktive Ausstattung mit Forschungsgeldern. Eigenverantwortliche Mittelverwendung, Personalressourcen und zur Verfügung gestellte Räumlichkeiten sollen Unabhängigkeit in der Forschung garantieren. Gleichzeitig können die Wissenschaftler Erfahrungen im Bereich der Personalführung und -entwicklung sammeln. Die Einbindung in den Cluster mit der Möglichkeit, sowohl auf die Expertise der beteiligten Seniorwissenschaftler als auch die Ressourcen und Servicefunktionen des Clusters zurückgreifen zu können, soll die wissenschaftlichen Karrierewege der Nachwuchsgruppenleiter unterstützen, so die Ansicht der Clustersprecher. Offen ist, wie gut eine Austarierung zwischen übergeordneten Interessen des Clusters als Gesamtprojekt und möglicherweise (in Teilen) anders gelagerten inhaltlichen Zielvorstellungen der Nachwuchsgruppenleiter in der Praxis gelingt. $^{2}$

Während die Nachwuchsgruppenleiter für die Zeit ihrer Arbeit im Exzellenzcluster gute Forschungsbedingungen vorfinden, werden sich möglicherweise am Ende der Förderung Probleme einstellen: Denn wenn zu diesem Zeitpunkt der ausgebildete Nachwuchs auf den akademischen Arbeitsmarkt drängt und neue Karrierechancen sucht, muss sich zeigen, inwieweit das deutsche Wissenschaftssystem auch tatsächlich in der Lage ist, hierfür genügend attraktive Stellen zur Verfügung zu stellen. Die mittlerweile seit über einem Jahrzehnt im Wesentlichen stagnierende Gesamtzahl der Professuren in Deutschland (Statistisches Bundesamt 2008) lässt Zweifel aufkommen, dass das wirklich gelingen kann.

\subsection{TENURE TRACK}

Dieser absehbare Engpass betrifft nicht alle Postdocs, denn zumindest für einen Teil der eingestellten Wissenschaftler in den Exzellenzprojekten wurden Tenure TrackModelle entwickelt. Die Idee an sich ist zwar im deutschen Hochschulsystem nicht neu, durch die Exzellenzinitiative rückt sie jedoch stärker in den Blickpunkt. Verstanden wird hierunter im US-amerikanischen Original ein bereits bei der Einstellung eröffnetes Angebot, an der gleichen Einrichtung nach unterschiedlichen Erprobungsstufen in befristeten Anstellungsverhältnissen (Assistant Professor, Associate Professor) am Ende nach positivem Durchlaufen einer Evaluation auf eine unbefristete Position als Professor (Full Professor) zu rücken, ohne Hausberufungsverbote und in der Regel auch ohne Ausschreibungspflichten (Wissenschaftsrat 2005). Für den einzelnen Wissenschaftler bedeutet dieses eine klare Karriereperspektive, keineswegs jedoch einen Automatismus. So ist es in den renommiertesten US-amerikanischen Universitäten zwar üblich, Stellen mit Tenure-Optionen auszuschreiben, „diese aber letztlich selten in unbefristete Stellen umzuwandeln" (Herkommer 2007).

Zunächst ist einschränkend festzuhalten, dass nicht alle im Kontext der Exzellenzinitiative eingestellten Nachwuchsgruppenleiter sowie (Junior-)Professoren mit Tenure-Optionen versehen bzw. für zu verstetigende Stellen vorgesehen sind. Die erste zentrale Entscheidung, die Schulen und Cluster zu treffen haben, liegt damit in der Frage, ob überhaupt Verstetigungsoptionen geschaffen werden. Für die Attraktivität der Stellen und damit auch das Bewerberfeld spielen entsprechende Angebote aus Sicht der interviewten Sprecher eine zentrale Rolle. Gleichwohl werden auch Motive genannt, „exzellenzfinanzierte“ Professoren und Nachwuchsgruppenleiter nur befristet einzustellen. Ein pragmatisches Argument gegen Verstetigungen ist, dass die Gewährleistung einer langfristigen Finanzierung (die nicht aus Exzellenzgeldern geschehen kann) vor allem der Professuren schon in der Startphase der Projekte zu

\footnotetext{
2 Hierzu und zu weiteren Fragestellungen führt das iFQ in Kürze eine Onlinebefragung aller bis dato von Graduiertenschulen und Exzellenzclustern eingestellten Nachwuchsgruppenleiter durch.
} 
grundlegenden Konflikten geführt hätte. Auslöser können hier beispielsweise mit den Verstetigungen einhergehende Befürchtungen sein, es käme zukünftig zu personellen Verschiebungen innerhalb von oder zwischen Fachbereichen und Instituten - ohne jedoch vorab zu wissen, welche Positionen davon betroffen sein werden. In diesen Fällen kann der Verzicht auf Entfristungen als Instrument intraorganisationaler Friedenssicherung verstanden werden, denn die getroffene Entscheidung antizipiert das Störpotenzial möglicher Veto-Spieler. Einige Sprecher sehen das Fehlen von Verstetigungsoptionen aus einer eher selbstbewusst-optimistischen Perspektive: Gerade für Juniorprofessoren und Nachwuchsgruppenleiter wird argumentiert, dass diese durch die überdurchschnittlich guten Forschungsbedingungen im Exzellenzprojekt schnell genügend Reputation erwerben können, um sicher bis zum Ende der Förderung einen (externen) Ruf auf eine Lebenszeitprofessur zu erhalten.

Für Verstetigungsoptionen sprechen aus Sicht der Interviewten neben einer deutlich höheren Attraktivität der Stellen auf potenzielle Bewerber und entsprechend besseren Auswahlmöglichkeiten seitens der Exzellenzeinrichtung auch langfristige Entwicklungsperspektiven: Diejenigen Kandidaten, die erfolgreich einen Tenure durchlaufen haben, sollen dauerhaft an die Hochschule gebunden werden und zur Entwicklung des Forschungsbereichs beitragen. Hier spielen also Aspekte längerfristiger Mitarbeiterentwicklung eine Rolle, wie man sie sonst bis dato eher in der außeruniversitären Forschung kannte (Böhmer/Hornbostel 2009).

Die Umsetzung der Verstetigungsmodelle verläuft nach unseren bisherigen Erkenntnissen sehr variantenreich. Den Tenure an exzellenzgeförderten Hochschulen gibt es (noch) nicht. Vielmehr sind unterschiedliche Verfahren entstanden, in denen sich auch Berufungskulturen der Universitäten, Strategien der Graduiertenschulen und Exzellenzcluster, hochschulrechtliche Rahmenbedingungen der Länder, Ausnahmen im Hausberufungsverbot und nicht zuletzt die zweifelsohne notwendigen Flexibilitäten in den Hochschulhaushalten für die kommenden Jahre widerspiegeln. Ein erster Gliederungsversuch lässt eine Unterscheidung zwischen Tenure Track und Tenure Option sinnvoll erscheinen.
(1) Tenure Track bedeutet hier, dass die Entfristung der (Junior-)Professur oder die Berufung eines Nachwuchsgruppenleiters auf eine Professur an ein personenbezogenes Evaluationsverfahren gekoppelt ist. Eine Ausschreibung findet in diesem Fall nicht statt, vielmehr werden individuelle Leistungen bewertet, wobei (auch) auf externe Gutachter zurückgegriffen wird. Die Entfristung der Professur kann dabei mit einem Aufstieg in der W-Besoldung verbunden sein. Bei Juniorprofessuren (W1) ist dies zwangsläufig der Fall: W1Positionen sind von ihrer Grundanlage her befristet und können in dieser Qualität nicht verstetigt werden. Ein interessanter Befund unserer Interviews ist, dass obwohl eine ganze Reihe solcher Tenure Tracks geplant ist, die Bewertungsverfahren zur Zeit noch nicht überall im Detail feststehen. Hier sei man aktuell noch dabei, sowohl das Verfahren als auch die maßgeblichen Bewertungskriterien auszuformulieren, so die interviewten Sprecher.

(2) Von einer Tenure Option ist hingegen dann zu sprechen, wenn zwar eine Verstetigung im Grundsatz möglich ist, diese aber nicht in Form eines auf eine Person zugeschnittenen Verfahrens stattfindet, sondern im Wettbewerb mit anderen Kandidaten. Wettbewerb muss nicht zwingend mittels einer externen Ausschreibung hergestellt werden, sondern kann auch darüber erfolgen, dass für eine Anzahl interner Kandidaten eine geringere Zahl an Dauerstellen eingeplant wird. Ein Exzellenzcluster, der bspw. zehn Juniorprofessuren einrichtet, hält für diesen Personenkreis - in Vereinbarung mit der Hochschulleitung und den betroffenen Fakultäten - fünf Lebenszeitprofessuren „reserviert“. Dieses in der Praxis oft „Competitive Tenure“ genannte Modell führt zwangsläufig zu projektinterner Konkurrenz. Auch hier sind die Verfahren noch zu neu, als dass deren Effekte schon bewertbar wären. Die Unterschiede zum Tenure Track werden aber bereits deutlich: Erhöhte Konkurrenz geht einher mit verminderter individueller Planungssicherheit. Inwiefern die betroffenen Nachwuchsgruppenleiter und Juniorprofessoren ihre Karriereplanungen und ggf. auch Forschungsstrategien daran anpassen (Kann man sich "Risikoforschung“ unter diesen Umständen leisten? Muss man sie im Gegenteil zwangsläufig anstreben?), ist noch nicht abschätzbar.
Der Tenure wurde in vielen Fällen von Anfang an mitgedacht und entsprechende Planungen wurden bereits in den Förderanträgen niedergelegt. Ein Teil der befristeten „Exzellenzprofessuren“ sollte in jedem Fall verstetigt werden. Vor allem, um (international) wettbewerbsfähige Angebote an herausragende Bewerber machen zu können, wurden aber auch nach Beginn der Exzellenzförderung in Abstimmung mit den Fakultäten und der Hochschulleitung noch weitere Verstetigungen möglich gemacht, die im Förderantrag nicht eingeplant waren. Hier zeigt sich ein Lerneffekt der Exzellenzprojekte, was die Wirksamkeit solcher Nachhaltigkeitsperspektiven auf die Qualität der Bewerberpools anbelangt.

$\mathrm{Ob}$ aber von Beginn an oder im Rahmen der Einstellungsverfahren später zugesagt: Die Darstellung dieser Tenure aus den bestehenden Haushalten stellt die betroffenen Hochschulen aktuell und in Zukunft - wenn nämlich die Exzellenzförderung ausläuft - vor Herausforderungen, so die Äußerungen der von uns interviewten Hochschulkanzler. Je mehr solcher Zusagen bei konstanter oder nicht reziprok steigender (Grund-)Finanzierung gemacht worden sind, desto stärker schränkt dieses den Handlungsspielraum der Hochschulen für die demnächst anstehende neue Auswahlrunde der Exzellenzinitiative ein. Gemäß den Ausschreibungsunterlagen erwarten DFG und Wissenschaftsrat sowohl von Neu- als auch von Fortsetzungsanträgen Aussagen zur Nachhaltigkeit: „Dabei ist in Fortsetzungsanträgen auszuführen, inwiefern die seinerzeit in den Neuanträgen gegebenen Zusagen umgesetzt worden sind.“ (DFG/WR 2010).

\section{4 \\ Lerneffekte?}

Die Anwendung des Tenure im Kontext der Exzellenzinitiative lässt sich aktuell also zusammenfassend als Experimentierfeld beschreiben - was durchaus den Programmzielen entspricht: In Abhängigkeit von den lokalen Bedingungen und den strategischen Zielen sollten die Exzellenzeinrichtungen die für sich passenden Maßnahmenbündel schnüren. Unkonventionelle Ideen wurden ausdrücklich begrüßt und die Initiative wurde insgesamt als „Ideenwettbewerb für Forschung und Strategien" beschrieben (Winnacker 2005), 
begrenzt lediglich durch hochschulrechtliche Rahmenbedingungen. Diese Experimentierfreude führt gleichwohl dazu, dass - soweit überhaupt ein Tenure angeboten wird - einige Unklarheiten bestehen bleiben. Insofern überrascht es nicht, dass nach Einschätzung der Interviewten ein externer Ruf für die Postdocs und befristet eingestellten (Junior-)Professoren bessere Möglichkeiten bietet: Neben der sicheren Stelle eröffnet dieser Ruf nämlich die Möglichkeit für Bleibeverhandlungen, im Fall der Nachwuchsgruppenleiter ggf. auch für vorgezogene Tenure-Verfahren. Sprecher von Exzellenzclustern berichteten in unseren Interviews, dass es einer Reihe von Wissenschaftlern in ihrem Cluster auf diesem Weg bereits gelungen sei, sich eine Verstetigung an der Hochschule zu sichern.

Der Start der Exzellenzinitiative fiel in eine Zeit, die insgesamt von intensiven Reformanstrengungen in der deutschen Hochschullandschaft geprägt war (und es weiterhin ist). Nicht alle geschilderten Entwicklungen sind also auf die Exzellenzinitiative allein zurückzuführen. Mit Tenure-Modellen wurde schon früher experimentiert. Gleichzeitig kann die Exzellenzinitiative als befristetes Drittmittelprogramm nicht alle Probleme lösen, die sich teilweise seit Jahrzehnten in deutschen Hochschulen zeigen. Stellen lassen sich damit zwar einrichten, nicht jedoch über 2017 hinaus finanzieren. Den auf der einen Seite mit Engagement vorangetriebenen Konzepten zur Verbesserung der Situati- on von Nachwuchswissenschaftlern fehlt es auf der anderen Seite aus Gesamtperspektive an zahlenmäßig ausreichenden und tragfähigen Zukunftschancen in der deutschen Wissenschaft. Die im Vergleich wenigen Verstetigungen im Kontext der Exzellenzinitiative lösen dieses Problem nicht. Möglicherweise treten am Ende der Förderung neue Probleme durch das Heer „exzellent" qualifizierter Nachwuchswissenschaftler auf, die die Aufnahmefähigkeit des deutschen Wissenschaftssystem überfordern könnten.

Ob Verstetigungen vorwiegend aus dem Bestand, d.h. durch Umschichtungen, dargestellt werden oder wirklich einen Nettogewinn an neuen Professuren bedeuten, der dann zwangsläufig zusätzliche Investitionen der Länder bedeuten würde, muss am Ende der Exzellenzförderung untersucht werden. Interviews mit Hochschulkanzlern bestätigen im Moment eher die These, dass Verstetigungen derzeit vorwiegend durch Umschichtungen stattfinden bzw. so geplant sind. Damit wirkt die Exzellenzinitiative an den geförderten Hochschulen zwar Profil bildend - für das Wissenschaftssystem insgesamt bliebe es in einem solchen Fall jedoch bei einem Nullsummenspiel.

Gleichzeitig zeigt sich aber auch, dass vielerorts Erfahrungen mit Verstetigungsmodellen gesammelt und strukturierte Karrierepfade von der Promotion über die Postdocphase bis hin zur Professur entwickelt und umgesetzt werden, die durchaus beispielhaft für das deutsche Hochschul- system sein können. Die Exzellenzinitiative macht darüber hinaus deutlich, dass vergleichsweise schnell erfolgreiche Lösungsansätze für Personalrekrutierungen gefunden werden konnten, die Ansätze systematischer Karrierewege beinhalten und dem Anspruch der Nachwuchswissenschaftler auf eine gewisse Planungssicherheit und erkennbare Karrierechancen gerecht werden. Die konkrete Ausgestaltung zeigt sich als variantenreiches Patchwork US-amerikanischer Schnittmuster mit jeweils lokal unterschiedlichen hochschulund projektspezifischen Ausgangslagen und Zielhorizonten - die teilweise auch nach wie vor das Beharrungsvermögen der Kameralistik und den traditionellen Status des Professors erkennen lassen. Für das deutsche Hochschulsystem wird es wichtig sein, dass positive wie negative Erfahrungen aus dem Kontext der Exzellenzinitiative publik werden und eine breite Partizipation an Lerneffekten ermöglichen. Nur so kann die Exzellenzinitiative einen Beitrag leisten, damit im Gesamtsystem „eine kohärente Konzeption des Personalmanagements [entsteht], die eine Fülle von Anforderungen gleichzeitig zu bewältigen hätte: die Verantwortung gegenüber Promovierenden, Übergänge in nicht-universitäre Arbeitsfelder zu erleichtern, die reflektierte und von Themenkonjunkturen unabhängige Ausgestaltung von Stellenprofilen und eine Rekrutierungspolitik, die Fächern mittelfristig die erforderlichen Freiheitsgrade belässt“ (König 2010).

\section{LITERATUR}

Böhmer, S./Hornbostel, S. (2009): Postdocs in Deutschland: Vergleich von Nachwuchsgruppenleiterprogrammen, iFQ-Working Paper 6, Bonn (http://www.forschungsinfo.de/Publikationen/Download/working_ paper_6_2009.pdf)

Buchholz, K./Gülker, S./Knie, A./Simon, D. (2009): Attraktivität von Arbeitsbedingungen in der Wissenschaft im internationalen Vergleich: Wie erfolgreich sind die eingeleiteten wissenschaftspolitischen Initiativen und Programme?, Studie im Rahmen der Ausschreibung "Schwerpunktsicherung zu Forschung und Innovation in Deutschland ", Expertenkommission Forschung und Innovation der Bundesregierung (EFI),Berlin Bundesanzeiger (2009): Verwaltungsvereinbarung zwischen Bund und Ländern gemäß Artikel 91b Abs. 1. Nr. 2 des Grundgesetzes über die Fortsetzung der Exzellenzinitiative des Bundes und der Länder zur Förderung von Wissenschaft und Forschung an deutschen Hochschulen - Exzellenzvereinbarung II, Bundesanzeiger 103 vom 16.07., S. 2416
Deutsche Forschungsgemeinschaft (DFG)/Wissenschaftsrat (WR) (2008): Bericht der Gemeinsamen Kommission zur Exzellenzinitiative an die Gemeinsame Wissenschaftskonferenz (http://www.gwk-bonn.de/ fileadmin/Papers/GWK-Bericht-Exzellenzinitiative.pdf)

Deutsche Forschungsgemeinschaft (DFG)/Wissenschaftsrat (WR) (2010): Exzellenzinitiative des Bundes und der Länder zur Förderung von Wissenschaft und Forschung an deutschen Hochschulen. Ausschreibung für die zweite Programmphase (http://www.dfg.de/download/ pdf/foerderung/programme/exin/ausschreibung_2_programmphase.pdf) Enders, J. (1996): Die wissenschaftlichen Mitarbeiter. Ausbildung, Beschäftigung und Karriere der Nachwuchswissenschaftler und Mittelbauangehörigen an den Universitäten, Frankfurt/Main

Gülker, S./Böhmer, S. (2009): Nachwuchspolitik, in: Simon, D./ Knie, A./ Hornbostel, S. (Hrsg.): Handbuch Wissenschaftspolitik, Wiesbaden, S. $176-192$ 
Hartmann, M. (2006): Die Exzellenzinitiative - ein Paradigmenwechsel in der deutschen Hochschulpolitik, in: Leviathan 4, S. 447-465 Herkommer, V. (2007): Tenure Track im deutschen Wissenschaftssystem: Das Verhältnis der Juniorprofessoren zu drittmittelgeförderten Nachwuchswissenschaftlern, iQ-Beitrag (http://www.forschungsinfo. de/iq/agora/Tenure_Track/tenure_track.asp)

Hornbostel, S./Simon, D./Heise, S. (Hg.) (2008): Exzellente Wissenschaft. Das Problem, der Diskurs, das Programm und die Folgen, iFQWorking Paper 4, Bonn (http://www.forschungsinfo.de/Publikationen/ Download/working\%20paper_4_2008.pdf)

Janson, K./Schomburg, H./Teichler, U. (2007): Wege zur Professur. Qualifizierung und Beschäftigung an Hochschulen in Deutschland und USA, Münster

König, M. (2010): Nachwuchsförderung im Rahmen der Exzellenzinitiative, in: Leibfried, S. (Hrsg.): Die Exzellenzinitiative: Erkundungen, Kontexte, Empfehlungen, Frankfurt/Main, S. 157-172

Markschies, C. (2007): Die Exzellenz und der Exzellenz-Wettbewerb, in: Gegenworte 17, S. 17-22

Münch, R. (2007): Die akademische Elite, Frankfurt/Main

Pasternack, P. (2008): Die Exzellenzinitiative als politisches Programm Fortsetzung der normalen Forschungsförderung oder Paradigmenwechsel, in: Bloch, R./Keller, A./Lottmann, A./Würmann, C. (Hrsg.): Making Exzellence, Reihe GEW Materialien aus Hochschule und Forschung 114, Bielefeld, S. 13-36
Simon, D./Knie, A. (2007): Aus Mangel an Beweisen: 'Exzellente' Kriterien in der Wissenschaft?, in: Gegenworte 17, S. 27-29

Sondermann, M./Simon, D./Scholz, A.-M./Hornbostel, S. (2008): Die Exzellenzinitiative: Beobachtungen aus der Implementierungsphase, iFQ-Working Paper 5 (http://www.forschungsinfo.de/Publikationen/ Download/working_paper_5_2008.pdf)

Sozialdemokratische Partei Deutschlands (SPD) (2004): Unser Land gerecht erneuern - Weimarer Leitlinien Innovation, Pressemitteilung vom 06.01 .

Statistisches Bundesamt (2008): Bildung und Kultur: Personal an Hochschulen. Vorläufige Ergebnisse, Selbstverlag

Von Münch, I. (2005): Elite-Universitäten. Leuchttürme oder Windräder?, Hamburg

Winnacker, E.-L. (2005): Entstehung und Ziele der Exzellenzinitiative, in: Forschung 02

Wissenschaftsrat (WR) (2005): Empfehlungen zur Ausgestaltung von Berufungsverfahren (http://www.wissenschaftsrat.de/texte/6709-05. pdf) 